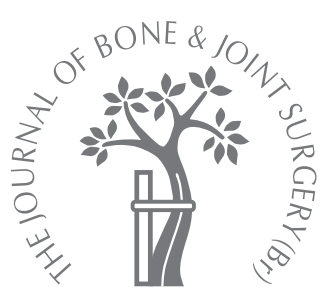

E. K. Shin, N. F. Jones, J. F. Lawrence

From The UCLA Centre for Health Sciences, Los Angeles, USA

\title{
Treatment of multiple hereditary osteochondromas of the forearm in children
}

\author{
A STUDY OF SURGICAL PROCEDURES
}

E. K. Shin, MD. Resident Department of Orthopaedic Surgery

UCLA, Centre for Health Sciences, 10833 LeConte Avenue, Los Angeles 90095 California, USA.

N. F. Jones, MD, Professor and Director

UCLA Hand Centre

UCLA Centre for Health

Sciences, 200 UCLA Medical

Plaza 140, Los Angeles

90020, California, USA.

J. F. Lawrence, MD,

Director

Hand Surgery Service

Shriners Hospital of Los

Angeles, 3160 Geneva

Street, Los Angeles 90095,

California, USA

Correspondence should be sent to Dr E. K. Shin; e-mail: eshin@mednet.ucla.edu

(C)2006 British Editorial

Society of Bone and

Joint Surgery

doi:10.1302/0301-620X.88B2.

$16794 \$ 2.00$

$J$ Bone Joint Surg [Br]

2006;88-B:255-60.

Received 31 May 2005

Accepted after revision

7 September 2005

We have evaluated the clinical outcomes of simple excision, ulnar lengthening and the Sauvé-Kapandji procedure in the treatment of deformities of the forearm in patients with multiple hereditary osteochondromas. The medical records of 29 patients (33 forearms) were reviewed; 22 patients ( 22 forearms) underwent simple excision (four with ulnar lengthening) and seven the Sauvé-Kapandji procedure.

Simple excision increased the mean supination of the forearm from $63.2^{\circ}$ to $75.0^{\circ}$ ( $p=0.049$ ). Ulnar lengthening did not significantly affect the clinical outcome. The SauvéKapandji technique improved the mean pronation from $33.6^{\circ}$ to $55.0^{\circ}(p=0.047$ ) and supination from $70.0^{\circ}$ to $81.4^{\circ}(p=0.045)$. Simple excision may improve the range of movement of the forearm but will not halt the progression of disease, particularly in younger patients. No discernable clinical or radiological improvement was noted with ulnar lengthening. The Sauvé-Kapandji procedure combined with simple excision of osteochondromas can improve stability of the wrist, movement of the forearm and the radiological appearance.

Multiple hereditary osteochondromas occur as a result of a disorder of enchondral bone growth characterised by metaphyseal protrusions of cartilage-capped bone. ${ }^{1-3}$ Considerable deformities of the forearm occur in $30 \%$ to $60 \%$ of patients with this disease. ${ }^{4}$ The most common are relative shortening of the ulna, bowing of either or both of the forearm bones, ulnar tilt of the distal radial epiphysis, ulnar deviation of the hand and dislocation of the head of the radius. They are usually associated with a discrepancy in length between the radius and ulna. Ulnar shortening probably occurs because the distal ulnar physis contributes more to total length than the distal radial physis does to the total length of the radius. In addition, the distal ulnar physis has a smaller cross-sectional area than that of the distal radius, causing a greater proportional involvement of the ulnar growth plate. ${ }^{5}$

A number of techniques for the surgical management of deformities of the forearm have been described. ${ }^{6-11}$ Surgical intervention is indicated for pain or a functional deficit related to an osteochondroma. Occasionally, surgery is performed to improve the cosmetic appearance.

Some studies have shown a more significant improvement in appearance than in function after surgery. ${ }^{12-14}$ Stanton and Hansen ${ }^{14}$ found that patients with multiple hereditary osteochondromas tolerated deformities of the upper limb well and had little loss of function measured both subjectively and objectively. Arms et $\mathrm{al}^{12}$ suggested that correction of deformity and improvement of function were not necessarily associated. Known surgical interventions include simple excision of osteochondromas, acute or gradual ulnar lengthening, corrective radial osteotomy, hemi-epiphyseal stapling of the distal radius, creation of a one-bone forearm ${ }^{10}$ and the Sauvé-Kapandji procedure. ${ }^{7}$

The most common operation performed in children is simple excision. Fogel et $\mathrm{al}^{6}$ observed that excision of osteochondromas was not effective in controlling the progression of deformity in a series of ten patients. However, Masada et $\mathrm{al}^{8}$ demonstrated excellent functional results with simple excision in two patients as long as the procedure was limited to those with relative radial shortening secondary to distal osteochondromas of the radius. Wood et $\mathrm{al}^{11}$ demonstrated improved cosmesis after simple excision in four patients, but only minimal improvement in rotation of the forearm.

The rationale for ulnar lengthening is that the hypoplastic distal ulna tethers the radial physis, theoretically diminishes ulnar support for the carpus, and increases ulnar-sided pressure on the radial epiphysis. Fogel et $\mathrm{al}^{6}$ found 


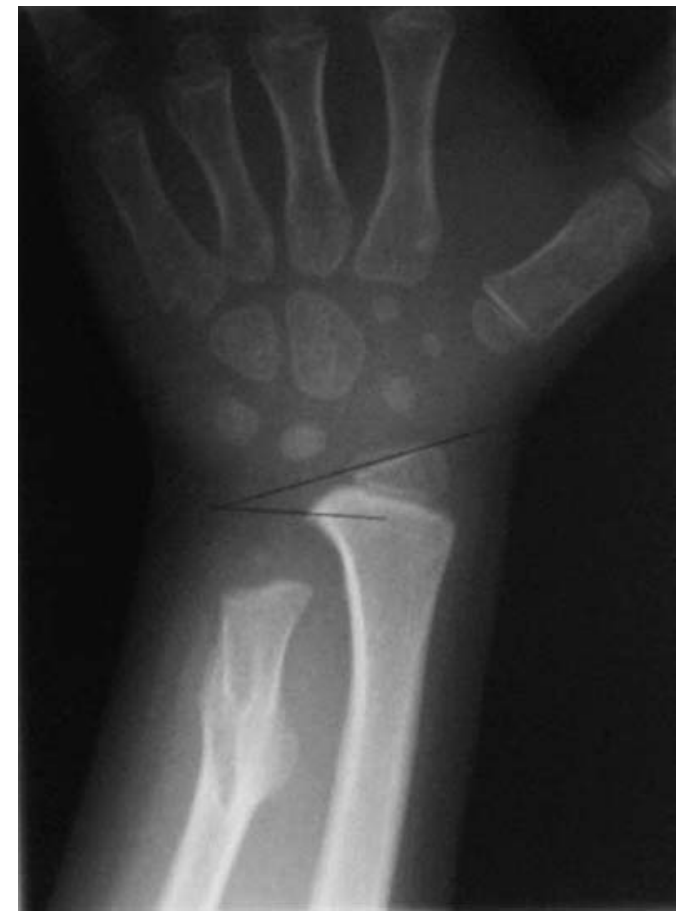

Fig. 1a

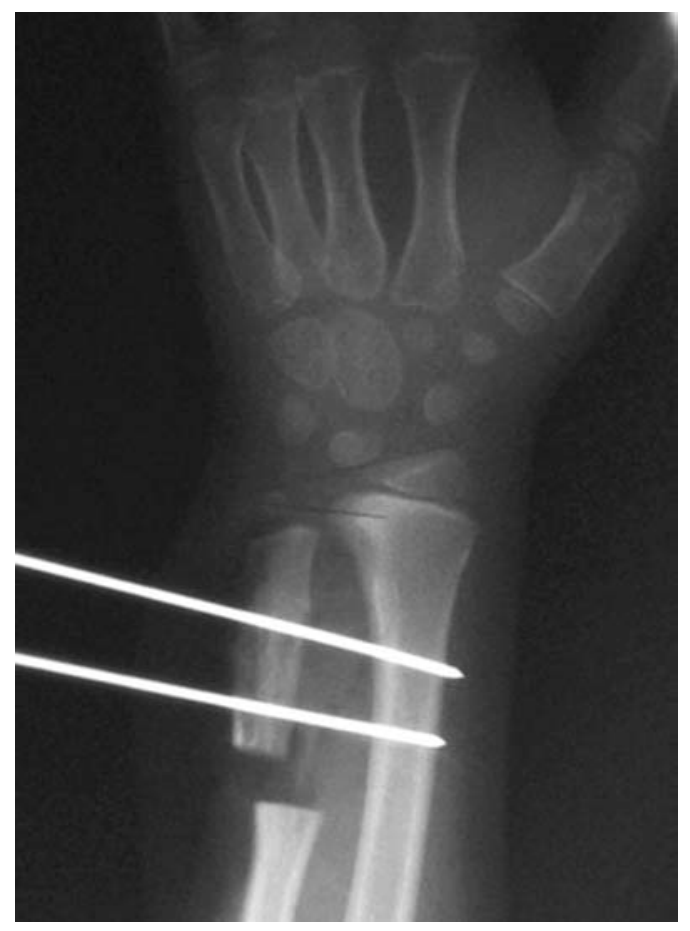

Fig. 1c

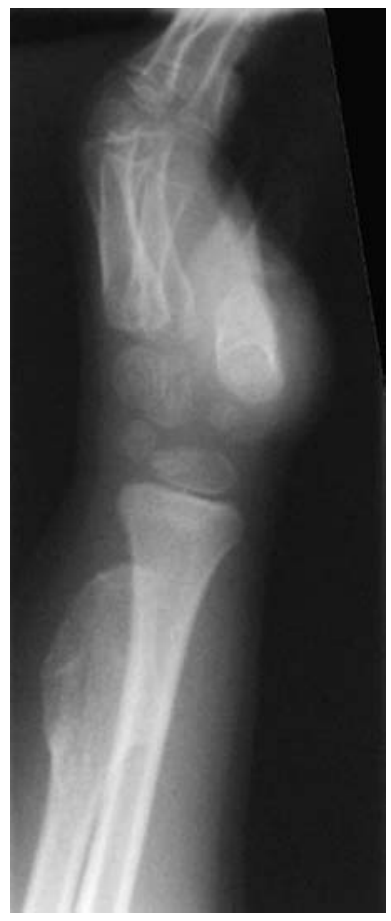

Fig. 1b

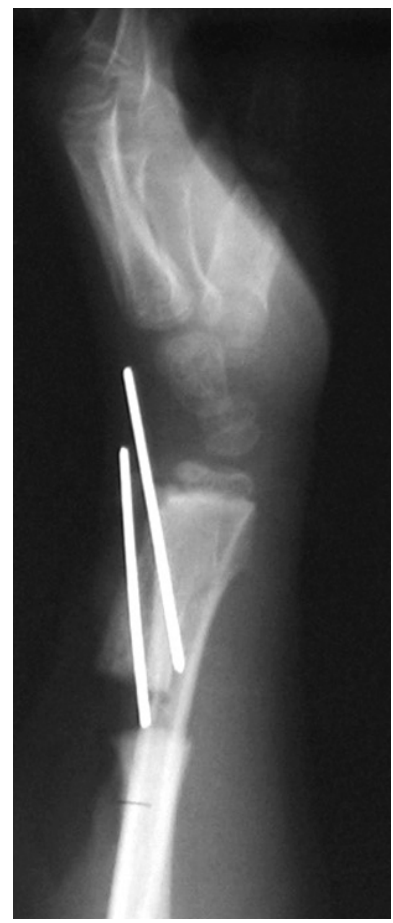

Fig. 1d

Radiographs showing the Sauvé-Kapandji procedure, modified for use in children to avoid injury to the distal ulnar physis, a) and b) pre-operative and c) and d) immediately post-operative. Fixation is achieved by two Kirschner wires instead of a single screw. 
that ulnar lengthening could sometimes correct ulnar drift at the wrist, but that relative shortening of the ulna tended to recur. Pritchett ${ }^{9}$ found that radial deviation at the wrist improved, as did stability of the radial head and rotation of the forearm. Masada et $\mathrm{al}^{8}$ in a study of four patients concluded that ulnar lengthening was effective in patients with relative ulnar shortening and dislocation of the radial head.

The Sauvé-Kapandji procedure involves fusion of the distal radioulnar joint combined with the intentional formation of a distal ulnar pseudarthrosis to allow pronation and supination of the forearm. It is usually used for pain in the wrist secondary to traumatic or rheumatoid arthritis. Although a stable distal radioulnar joint with ulnocarpal support is achieved by this technique, the potential for an unstable proximal ulna remains.

Our aim was to compare the clinical and radiological results in the forearm of three operations, namely, simple excision of osteochondromas, ulnar lengthening with excision of osteochondromas, and the Sauvé-Kapandji procedure with excision of osteochondromas.

\section{Patients and Methods}

Between 1982 and 2001, 45 patients between the ages of four and 19 years with multiple hereditary osteochondromas underwent operation on the forearm at the Hospital for Children. The medical records of these patients were reviewed retrospectively with the approval of the hospital's institutional review board. Patients with clinical and radiological follow-up of less than 24 months were excluded from the study. Twenty-nine patients (33 forearms) were identified with a mean post-operative follow-up of 42.9 months (24 to 104). Twenty-two patients (22 forearms) had undergone simple excision of osteochondromas (four with ulnar lengthening), and seven had the Sauvé-Kapandji procedure with excision.

Of the four patients who underwent ulnar lengthening, it was performed in two by gradual distraction with an external fixator, and in the other two by an immediate bone graft and step-cut osteotomy with internal fixation. Lengthening was $20 \mathrm{~mm}$ or less to prevent potential neurovascular problems.

The original Sauvé-Kapandji procedure was modified for children by using a longer distal fragment of the ulna. The ulnar shaft was typically transected 1.5 to $2.0 \mathrm{~cm}$ proximal to the distal end and fixed to the radius at an angle. The angulated position was chosen in order to maintain adequate distance between the radial and ulnar shafts to facilitate rotation of the forearm. Fixation was achieved by two transverse Kirschner wires (Fig. 1).

Clinical evaluation. The indications for surgery included pain, functional loss of movement of the forearm and cosmesis. Pronation, supination and flexion and extension of the elbow were recorded immediately before operation and at the last post-operative visit. Any recurrence requiring further surgery and any surgical complications were noted.
Radiological evaluation. Anteroposterior radiographs of each forearm were examined to determine the location of the osteochondromas and the presence of subluxation or dislocation of the radial head. Figure 2 shows the parameters measured before and after operation as described by Burgess and Cates. ${ }^{15}$

Statistical analysis. The mean and SDs for all values were calculated from pre-operative clinical and radiological measurements. These were then compared with calculated mean values obtained after operation. Statistical comparisons between pre-operative and post-operative ranges of movement and radiological parameters were performed using a two-tailed Student's $t$-test. A p value of $\leq 0.05$ was considered to be statistically significant for all tests.

\section{Results}

Excision of osteochondromas. The mean age of the 22 patients who underwent simple excision of osteochondromas was was 9.2 years. The indication for surgery was limitation of movement in eight, pain in seven, cosmesis in six, and pain and limitation of movement in one. Deformity at the distal end of both the radius and ulna was present in 11 forearms. Six had osteochondromas at the distal ulna and five at the distal radius. No patient required excision of a proximal radial or ulnar osteochondroma. Dislocation of the radial head was not present in any of these forearms at the time of surgery.

Simple excision of osteochondromas led to a significant increase in mean supination of the forearm from $63.2^{\circ}$ to $75.0^{\circ}(\mathrm{p}=0.049)$, but did not improve pronation or flexion and extension of the elbow significantly. The radiological parameters did not change significantly.

Simple excision alone resulted in a rate of recurrence requiring further surgery of $36.4 \%$. In patients aged ten years or older at the time of operation, the rate of recurrence was $11.0 \%$. In patients younger than ten years, it was $53.8 \%$. All patients with recurrence were treated by repeated simple excision.

Ulnar lengthening with excision of osteochondromas. The mean age of the four patients who underwent ulnar lengthening with excision of osteochondromas was 8.8 years. Two underwent surgery for dislocation of the radial head, one for pain and one for cosmesis and pain. All had osteochondromas in the distal ulna only.

Ulnar lengthening did not significantly affect the clinical outcome. Pronation remained unchanged while the mean supination improved from $82.5^{\circ}$ to $90.0^{\circ}$. The mean flexion of the elbow improved from $122.5^{\circ}$ to $133.8^{\circ}$ while the mean extension showed a small improvement from $2.5^{\circ}$ to $0.0^{\circ}$. However, statistical significance was not achieved in any of these clinical findings. The radiological parameters also did not change significantly with ulnar lengthening. Further surgery was required re-operation in two patients for loss of movement. These patients showed persistent dislocation of the radial head, subsequently requiring open reduction and reconstruction of the annular ligament. 

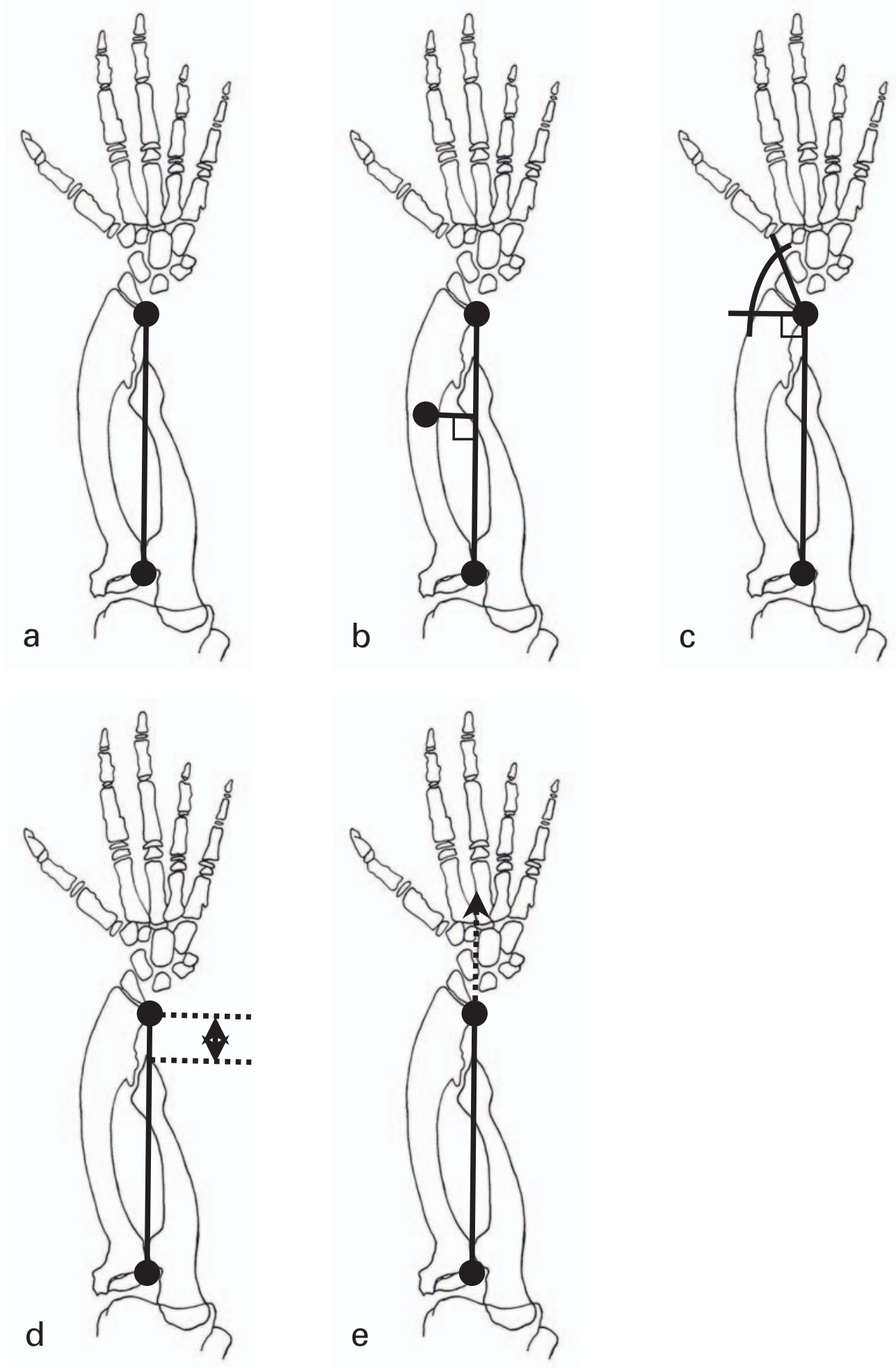

Fig. 2

Diagrams showing the radiological measurements. Figure 2a - Linear axis: a line drawn from the ulnar border of the distal and proximal radial physeal plates. Figure $2 b-$ Radial bow. the greatest distance that the radial diaphysis deviates from the linear axis of the forearm divided by the length of the linear axis. Figure 2c-Radial articular angle: the angle subtended by the inclination of the distal articular surface of the radius and the linear axis of the forearm. Figure $2 \mathrm{~d}$ - Ulnar variance: measured by a perpendicular line drawn from the distal end of the ulna to the linear axis of the forearm. Figure $2 \mathrm{e}$ - Carpal slip: the percentage of the lunate on the ulnar side of a continuation of the linear axis of the forearm. 
Another patient required washout of an infected pin-tract site and removal of hardware.

Sauvé-Kapandji procedure with excision of osteochondromas. Seven patients underwent the Sauvé-Kapandji procedure with excision of osteochondromas. The mean age at the time of operation was 11.1 years. Three underwent surgery for limitation of movement, two for pain and two for cosmesis. Deformities of the distal radius and ulna were present in four while two had deformities at the distal ulna only and one at the distal radius only. No osteochondromas were localised in the proximal radius or ulna.

The Sauvé-Kapandji procedure significantly improved the mean pronation from $33.6^{\circ}$ to $55.0^{\circ}(\mathrm{p}=0.047)$ and supination from $70.0^{\circ}$ to $81.4^{\circ}(\mathrm{p}=0.045)$. Flexion and extension of the elbow did not change. The mean radial articular angle improved from $46.0^{\circ}$ to $27.8^{\circ}(\mathrm{p}=0.023$ ). The mean percentage carpal slip also improved from $83.3 \%$ to $37.3 \%(p=0.031)$. No other significant changes were noted in the radiological measurements.

One patient required further surgery for recurrence four years and two months after the initial procedure. Simple excision of the recurrent osteochondroma was required. None of the complications sometimes associated with the Sauvé-Kapandji procedure such as nonunion or heterotopic ossification was observed in this group.

\section{Discussion}

Our study reports the results of three operations performed in patients with multiple hereditary osteochondromas of the forearm and shows that simple excision of osteochondromas can improve the range of movement of the forearm but will not halt the progression of disease, particularly in younger patients. Fogel et $\mathrm{al}^{6}$ described simple excision in ten patients and concluded that it was not effective in controlling progression of the deformity. Osteochondromas excised from mature patients did not recur, but in four forearms from which the lesions had been excised before puberty the results were variable and unpredictable.

By contrast, other series have shown that simple excision is effective in preventing progression of the disease and improving rotation. ${ }^{4,8}$ Moreover, it helped to control bowing of the radius. ${ }^{8}$ In our series, the mean supination improved by $11.8^{\circ}$, while pronation did not change appreciably. Pre-operatively, usually either pronation or supination was affected, with the movement in the other direction being fairly free. None of the patients had dislocation of the radial head which is typically associated with gross discrepancy in growth of the forearm bones. Half of the forearms in our series underwent excision of osteochondromas at the distal ends of both the radius and ulna. Radiological parameters were not significantly altered by excision.

Ulnar lengthening should theoretically improve ulnar translocation at the wrist, but no statistically significant clinical or radiological improvements were noted in our patients. Fogel et $\mathrm{al}^{6}$ performed ten ulnar lengthening procedures in eight patients. They found that relative shorten- ing of the ulna usually recurred after the lengthening and also, in agreement with our findings, that ulnar lengthening did not result in a significant improvement in rotation of the forearm, radial articular angle or carpal slip.

Masada et $a l^{8}$ noted recurrence of ulnar shortening after lengthening in 13 forearms. However, they showed significant improvement in rotation and radiological appearance. Nine of the 13 forearms also underwent radial osteotomy and it is possible that these improvements were secondary to the osteotomy.

Pritchett ${ }^{9}$ reported the results of ulnar lengthening in ten forearms (eight patients). He found that the deformity tended to recur in younger patients and that further lengthening may be required. He recommended initial overcorrection in skeletally immature patients.

The small number of patients who underwent ulnar lengthening by two different methods make it difficult to draw conclusions from our results. However, rotation of the forearm and movement of the elbow were slightly improved by ulnar lengthening.

The Sauvé-Kapandji procedure has been used to treat a variety of complex abnormalities of the distal radioulnar joint. Johnson et $\mathrm{al}^{7}$ described ten patients treated by this procedure. Most were in their early teens, the youngest being six years of age. Significant benefit was obtained in every case, although a second unplanned procedure was necessary in two patients because the rotation gap in the ulna filled in with bone. Three had multiple hereditary osteochondromas. The first showed limitation of movement of the forearm which improved from $90^{\circ}$ of combined pronation and supination to $160^{\circ}$. The second was treated prophylactically for a distal ulnar osteochondroma and ulnar shortening of more than $1 \mathrm{~cm}$. This patient did well after surgery and had no further evidence of ulnar shortening. The third had pain in the wrist on rotation, extension and radial deviation. The distal radioulnar joint was unstable and deformed, with a radial articular angle of $40^{\circ}$. A Sauvé-Kapandji procedure with concomitant closing wedge osteotomy of the distal radial metaphysis was performed reducing the angle of inclination to $20^{\circ}$. Twenty months after surgery, the patient's wrist was free from pain with $65^{\circ}$ of pronation and $70^{\circ}$ of supination. However, the radial physis continued to grow asymmetrically, increasing the angle of inclination to $50^{\circ}$. In retrospect, the authors conceded that an epiphysiodesis should have been performed at the same time as the radial osteotomy.

Our series has shown a significant improvement in rotation of the forearm after the Sauvé-Kapandji procedure. The mean supination increased by $11.4^{\circ}$ and pronation by $21.4^{\circ}$. Excision of osteochondromas from the distal end of the radius or ulna was performed at the same time. The radial articular angle and the carpal slip also improved. The changes in these radiological parameters may be secondary to displacement of the distal ulnar segment distally and fixation to the radius. This essentially mimics the outcome of 
ulnar lengthening by reducing the tethering effect of the distal ulna on the radial physis.

We conclude that the Sauvé-Kapandji procedure in conjunction with simple excision of osteochondromas is a useful method of treating deformity of the forearm in patients with multiple hereditary osteochondromas. It can improve stability of the wrist, movement of the forearm and the radiological appearance.

No benefits in any form have been received or will be received from a commercial party related directly or indirectly to the subject of this article.

\section{References}

1. Ogden JA. Multiple hereditary osteochondromata: report of an early case. Clin Orthop 1976;116:48-60.

2. Peterson HA. Multiple hereditary ostechondromata. Clin Orthop 1989;239:222-30.

3. Solomon L. Hereditary multiple exostosis. J Bone Joint Surg [Br]1963;45-B:292-304.

4. Shapiro F, Simon S, Glimcher MJ. Hereditary multiple exostoses: anthropometric, roentgenographic, and clinical aspects. J Bone Joint Surg [Am] 1979;61-A:815-24.

5. Solomon L. Bone growth in diaphyseal aclasis. J Bone Joint Surg [Br] 1961;43-B:700-16.
6. Fogel GR, McElfresh EC, Peterson HA, Wicklund PT. Management of deformities of the forearm in multiple hereditary osteochondromas. J Bone Joint Surg [Am] 1984;66-A:670-80.

7. Johnson MK, Lawrence JF, Dionysian E. The Kapandji procedure for the treatment of distal radioulnar joint derangement in young patients. Contemp Orthop 1995; 31:291-8.

8. Masada K, Tsuyuguchi Y, Kawai H, et al. Operations for forearm deformity caused by multiple osteochondromas. J Bone Joint Surg [Br] 1989;71-B:24-9.

9. Pritchett JW. Lengthening the ulna in patients with hereditary multiple exostoses. J Bone Joint Surg [Br] 1986;68-B:561-5.

10. Rodgers WB, Hall JE. One-bone forearm as a salvage procedure for recalcitrant forearm deformity in hereditary multiple exostoses. J Pediatr Orthop 1993;13:587-91.

11. Wood VE, Sauser D, Mudge D. The treatment of hereditary multiple exostosis of the upper extremity. J Hand Surg [Am] 1985;10-A:505-13.

12. Arms DM, Streker WB, Manske PR, Schoenecker PL. Management of forearm deformity in multiple hereditary osteochondromatosis. J Pediatr Orthop 1997;17: 450-4.

13. Peterson HA. Deformities and problems of the forearm in children with multiple hereditary osteochondromata. J Pediatr Orthop 1994;14:92-100.

14. Stanton RP, Hansen MO. Function of the upper extremities in hereditary multiple exostoses. J Bone Joint Surg [Am] 1996;78-A:568-73.

15. Burgess RC, Cates $\mathbf{H}$. Deformities of the forearm in patients who have multiple cartilaginous exostosis. J Bone Joint Surg [Am] 1993;75-A:13-18. 